\title{
Identification of Source-Affected Output Characteristics of a Four-Quadrant Cascaded H-Bridge Converter Cell
}

M. Petkovic and D. Dujic

This material is posted here with permission of the IEEE. Such permission of the IEEE does not in any way imply IEEE endorsement of any of EPFL's products or services. Internal or personal use of this material is permitted. However, permission to reprint / republish this material for advertising or promotional purposes or for creating new collective works for resale or redistribution must be obtained from the IEEE by writing to pubs-permissions@ieee. org. By choosing to view this document, you agree to all provisions of the copyright laws protecting it. 


\section{Identification of Source-Affected Output Characteristics of a Four-Quadrant Cascaded $\mathrm{H}$-Bridge Converter Cell}

\author{
$1^{\text {st }}$ Marko Petković \\ Power Electronics Laboratory - PEL \\ École Polytechnique Fédérale de Lausanne - EPFL \\ Lausanne, Switzerland \\ marko.petkovic@epfl.ch
}

\author{
$2^{\text {nd }}$ Dražen Dujić \\ Power Electronics Laboratory - PEL \\ École Polytechnique Fédérale de Lausanne - EPFL \\ Lausanne, Switzerland \\ drazen.dujic@epfl.ch
}

\begin{abstract}
The development and integration of power electronics equipment and converters for medium voltage ac and dc application has created different subsystem interactions that require proper investigation, understanding, description and estimation of global system stability through impedance-admittance measurements and identification. Four-quadrant Cascaded $\mathrm{H}$ Bridge topology features high output voltage resolution and high effective switching frequency which enables high-dynamic, high-fidelity voltage perturbation injection for medium voltage impedance and admittance measurement. This paper assesses the impact that the active converter stage on the input side has on the output side, where the injection of the perturbation signal is realized. Models of the input and output converters are developed as well as the model of the connected system. To validate the theoretical developments, a new method to measure the singlephase output dynamics in the $d q$-frame is proposed.
\end{abstract}

\section{INTRODUCTION}

The increase in energy demand combined with the advances in the field of power electronics has been a driving factor for the development of renewable energy system as well as extensive placement into service of power conversion devices. The interaction of different elements in the grid, on one side leads to the change in power network behaviour that is becoming increasingly complex and on the other side results in system instabilities [1], [2]. In order to overcome this issue and prevent future instabilities, an early identification and characterization of the present system and its future potential components would reduce the risk of encountering unpredictable behavior which would, in turn, provide a stable network with uninterrupted operation. Some of the criteria used to assess the stability are presented in [3] and they rely either on analysing the minor-loop gain or the passivity of the system. Whatever the criteria are, the knowledge of the impedance of the network being analysed is required.

Unfortunately, the problem of high-power and medium voltage (MV) impedance/admittance measurement and system identification had not yet been fully resolved. Measurements in MV application require devices capable of operating at an MV level and injecting a perturbation into an MV system, while at the same time having sufficiently wide bandwidth in order to characterize the impedance over a wide frequency range. These requirements combined are not easily fulfilled at the MV level and thus the research performed in this field is scarce and the devices developed for that purpose are few. The MV impedance estimators developed today have either limited bandwidth, up to $1 \mathrm{kHz}$ or feature an output side transformer in order to step-up the voltage to the MV levels, which in turn also limits the bandwidth. Still, the demand for such equipment is growing due to the need to support the development of recent medium voltage dc (MVdc) and medium voltage ac (MVac) applications, grid integration of renewable energy sources and storage devices, energy transmission and distribution in the MV range [4]-[6] and assure safe integration with the existing apparatus.

One of the topologies capable of having sufficiently high voltage output and bandwidth is based on Cascaded H-Bridge (CHB) with an active input element interfaced to a multiwinding transformer (MWT), presented in Fig. 1. This topology has already been considered in [7], where the feasibility of using a multilevel topology for perturbation injection was studied. The topology consists of step-down MV-MWT with 15 secondary phase-shifted outputs permitting to stack up to 5 cells per phase and thus effectively increase the output voltage levels and switching frequency allowing higher frequency voltage perturbation injection and impedance measurement. As a matter of fact, the presence of the MWT on the input side is one of the advantages of this topology. Having the MWT at the input means that there is no need to have a step-up transformer on the output side to elevate the voltage to MV level. As a result, the output stage high-frequency bandwidth is not limited by the output transformer. The CHB cells outputs are interfaced to a three-phase $L C$-type filter on which the voltage output is controlled. A single cell of the CHB converter is presented in Fig. 2. It consists of an Active Front End (AFE) as the input conversion stage and an $\mathrm{H}$-bridge (HB) inverter as the output conversion stage. It is taken as an assumption that the dynamics of a single-phase leg consisting of five cells can be represented by the dynamics of one cell, which is why the cell in Fig. 2 is represented with an $L C$-type filter at the 
output.

The initial study on the feasibility of this solution was performed in [7], where there was a need to superimpose a high-frequency small-signal in addition to the fundamental component. When this is performed in a closed-loop, there exist possible interactions of synchronization and closed control loops of AFE and HB. Our work seeks to reveal if the presence of an active input element, such as AFE, impacts the dynamics of the output element, the HB and subsequently the cascaded connection of multiple HBs.

The motivation for this is that for precise injection of perturbation signal into the device under test (DUT), one needs to ensure that the input side of the $\mathrm{CHB}$ does not in any way limit the output and that there are no other frequency components present that may impact the measurements. The methodology to respond to this question puts to use the statespace modelling and presents the model of the cell in the $d q$-frame. For the purposes of confirming the theoretical statespace model developed a method to measure the single-phase output characteristics and represent it in $d q$-frame is developed in this work.

The rest of the paper is organised as follows. Section II describes the main features of a single cell of the $\mathrm{CHB}$ converter. Section III provides the state-space model of the cell in the $d q$-frame. Section IV characterizes the source-affected dynamics of the cell output. Section V proposed a new method for measuring control-to-output characteristics in $d q$-frame that makes use of single-phase measurements together with a wideband pseudo-random binary sequence (PRBS) signal Finally, Section VII concludes the work.

\section{CASCADED H-BRIDGE CELL}

The MWT (c.f. Fig. 1) supplies three-phase isolated voltages from its secondary, low-voltage, side to the cell. Isolated threephase supply makes the cells and the dc-links largely independent from each other [8]. The input filter of the cell is the $L$-type filter with its parasitic resistance. The rectification of the input voltage is performed by the three-phase AFE, while the dc-link serves as an energy buffer from which the output stage, a single-phase $\mathrm{HB}$, is supposed to inject perturbation voltages into an unknown network or DUT. The AFE control system comprises a PLL that performs synchronisation, as it is essentially a grid-connected converter through the MWT. Apart from this, it also performs the cascaded control of the dc-link voltage through the input current control. On the output side the HB performs cascaded control of its output voltage through the control of the filter inductor current.

Ideally, for the best possible impedance identification of the DUT, the output stage should be completely decoupled from the input one and the presence of the active element with its control should not be a limiting factor for a wide-frequency band perturbation injection and impedance measurements.

\section{Single Cell Modelling}

\section{A. Active Front End Modelling}

Closed-loop modelling of the AFE (cf. Fig. 3a) follows the state-space modelling approach outlined in [9], [10] and the intermediate developments are not given in this work, but only the final open-loop and closed-loop models in the Synchronous Reference Frame (SRF) are presented. State-space averaged model is presented in Eq. (1), where $V_{\mathrm{dc}}, D_{d}^{\mathrm{AFE}}, D_{q}^{\mathrm{AFE}}, I_{\mathrm{gd}}$ and $I_{\mathrm{gq}}$ are steady-state values of dc-link voltage with duty cycles and currents represented in $d q$-frame. To obtain the open-loop dynamics, the states-space model given in Eq. (1) can be solved using Eq. (2).

$$
\mathbf{G}=\mathbf{C}(s \mathbf{I}-\mathbf{A})^{-1} \mathbf{B}+\mathbf{D}
$$

Subsequently the dynamics is represented by Eq. (3). State and output variables are given in the vector on the left hand side side, while the input variables are given in the right hand side vector of Eq. (3).

$$
\left[\begin{array}{c}
\tilde{\mathbf{i}}_{\mathrm{g}, \mathrm{dq}} \\
\tilde{v}_{\mathrm{dc}}
\end{array}\right]=\left[\begin{array}{ccc}
\mathbf{Y}_{\mathrm{in}, \mathrm{o}} & \mathbf{G}_{\mathrm{io}, \mathrm{o}} & \mathbf{G}_{\mathrm{ci}, \mathrm{o}} \\
\mathbf{T}_{\mathrm{oi}, \mathrm{o}} & -Z_{\mathrm{out}, \mathrm{o}} & \mathbf{G}_{\mathrm{co}, \mathrm{o}}
\end{array}\right]\left[\begin{array}{c}
\tilde{\mathbf{v}}_{\mathrm{g}, \mathrm{dq}} \\
\tilde{i}_{\mathrm{dc}} \\
\tilde{\mathbf{d}}_{\mathrm{dq}}
\end{array}\right]
$$

Direct link between the AFE and $\mathrm{HB}$ is the output impedance of the AFE and thus is the element that could influence the dynamics of the HB. For this reason the next important step is to find the closed-loop output impedance of the AFE. In the closed-loop the AFE is controlled in cascaded manner, with the inner loop being the input current control using proportional integral (PI) controllers in the SRF and the outer controller being the dc-link voltage PI controller [11]. Under the current control the output impedance is given as

$$
Z_{\mathrm{out}, \mathrm{cl}}^{\mathrm{gcc}}=-Z_{\mathrm{out}, \mathrm{o}}+\mathbf{G}_{\mathrm{co}, \mathrm{o}}\left(\mathbf{G}_{\mathrm{dec}}-\mathbf{G}_{\mathrm{PI}}^{\mathrm{i}}\right) \mathbf{G}_{\mathrm{io}, \mathrm{cl}}^{\mathrm{gcc}}
$$

While closing the dc-link voltage loops the output impedance becomes

$$
Z_{\mathrm{out}, \mathrm{cl}}^{\mathrm{dvc}}=-\left(\mathbf{I}+\mathbf{G}_{\mathrm{co}, \mathrm{cl}}^{\mathrm{gcc}} \mathbf{G}_{\mathrm{PI}}^{\mathrm{v}}\right)^{-1} Z_{\mathrm{out}, \mathrm{cl}}^{\mathrm{gcc}}
$$

where $\mathbf{G}_{\mathrm{dec}}, \mathbf{G}_{\mathrm{PI}}^{\mathrm{i}}$ and $\mathbf{G}_{\mathrm{PI}}^{\mathrm{v}}$ are the current controller decoupling matrix, current controller PI regulator and voltage controller PI regulator. AFE with parameters in Table I was simulated in PLECS and its analytical output impedance from Eq. (5) was compared to the impedance obtained from the frequency response (cf. Fig. 4). The result is presented in Fig. 4 and it shows good match. Several things can be noted in the impedance shape. The impedance magnitude has a low value in low frequency range due to a high gain of the PI controller in the same region. The breaking point at $35 \mathrm{~Hz}$ where the impedance has almost unity gain denotes the end of the closed-loop dc-link control bandwidth. 


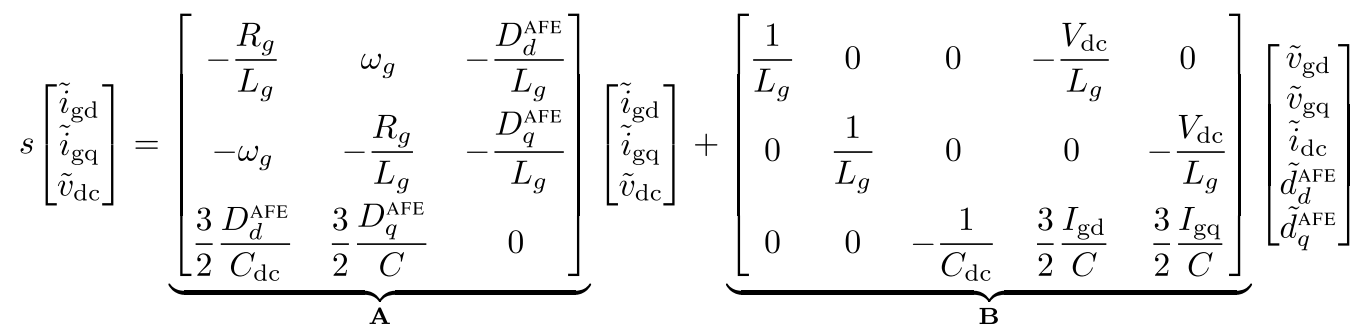

$$
\begin{aligned}
& {\left[\begin{array}{c}
\tilde{i}_{\mathrm{gd}} \\
\tilde{i}_{\mathrm{gq}} \\
\tilde{v}_{\mathrm{dc}}
\end{array}\right]=\underbrace{\left[\begin{array}{ccc}
1 & 0 & 0 \\
0 & 1 & 0 \\
0 & 0 & 1
\end{array}\right]}_{\mathbf{C}}\left[\begin{array}{c}
\tilde{i}_{\mathrm{gd}} \\
\tilde{i}_{\mathrm{gq}} \\
\tilde{v}_{\mathrm{dc}}
\end{array}\right]+\underbrace{\left[\begin{array}{ccccc}
0 & 0 & 0 & 0 & 0 \\
0 & 0 & 0 & 0 & 0 \\
0 & 0 & 0 & 0 & 0
\end{array}\right]}_{\mathbf{D}}\left[\begin{array}{c}
\tilde{v}_{\mathrm{gd}} \\
\tilde{v}_{\mathrm{gq}} \\
\tilde{i}_{\mathrm{dc}} \\
\tilde{d}_{d}^{\mathrm{AFE}} \\
\tilde{d}_{q}^{\mathrm{AFE}}
\end{array}\right]}
\end{aligned}
$$

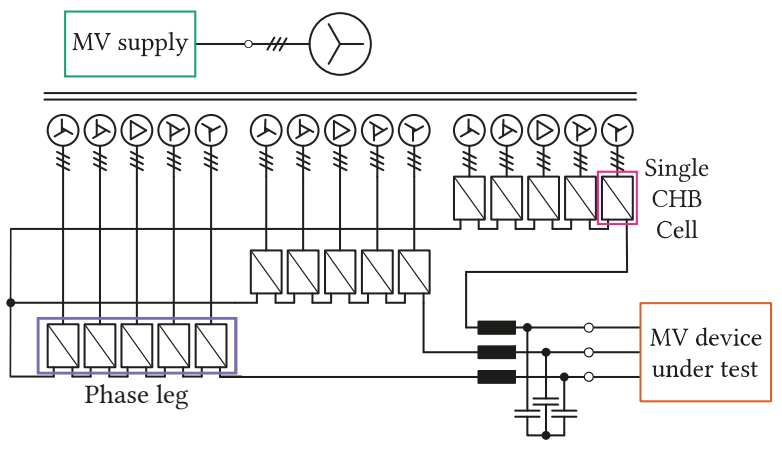

Fig. 1. Cascaded H-bridge topology for high dynamic mediumvoltage perturbation injection and impedance measurement.

TABLE I: AFE parameters

\begin{tabular}{ll}
\hline \hline$v_{\mathrm{g}, \mathrm{d}}=580 \mathrm{~V}$ & $f_{g}=50 \mathrm{~Hz}$ \\
$v_{\mathrm{g}, \mathrm{q}}=0 \mathrm{~V}$ & $L_{g}=5.4 \mathrm{mH}$ \\
$i_{\mathrm{g}, \mathrm{d}}=41.5 \mathrm{~A}$ & $R_{g}=10 \mathrm{~m} \Omega$ \\
$i_{\mathrm{g}, \mathrm{q}}=0 \mathrm{~A}$ & $v_{\mathrm{dc}}=1.2 \mathrm{kV}$ \\
$K_{\mathrm{p}, \mathrm{gcc}}=18$ & $K_{\mathrm{i}, \mathrm{gcc}}=33$ \\
$K_{\mathrm{p}, \mathrm{dvc}}=1.4$ & $K_{\mathrm{i}, \mathrm{dvc}}=192$ \\
\hline
\end{tabular}

TABLE II: HB parameters

\begin{tabular}{ll}
\hline \hline$v_{\mathrm{C}, \mathrm{d}}=600 \mathrm{~V}$ & $f_{s}=50 \mathrm{~Hz}$ \\
$v_{\mathrm{C}, \mathrm{q}}=0 \mathrm{~V}$ & $L_{f}=1.4 \mathrm{mH}$ \\
$i_{\mathrm{o}, \mathrm{d}}=60 \mathrm{~A}$ & $R_{f}=100 \mathrm{~m} \Omega$ \\
$i_{\mathrm{o}, \mathrm{q}}=0 \mathrm{~A}$ & $C_{f}=24 \mu \mathrm{H}$ \\
$K_{\mathrm{p}, \mathrm{lcc}}=5$ & $K_{\mathrm{i}, \mathrm{lcc}}=5$ \\
$K_{\mathrm{p}, \mathrm{cvc}}=1$ & $K_{\mathrm{i}, \mathrm{cvc}}=52$ \\
\hline
\end{tabular}

\section{B. Single-Phase Inverter Modelling}

The single-phase inverter injects precise perturbations into a DUT, imposing the requirement that the output voltage control should not be limited by the control loops of the AFE. i.e. by the closed-loop interaction of the AFE and HB. In order to find the closed-loop model, first the open-loop model and dynamics have to be defined and solved. As the dc-link voltage control is performed on the AFE side, the inverter input is initially treated as an ideal voltage source (cf. Fig. 3b). The modelling is performed in the $d q$-frame and it follows similar approach as the modelling of the AFE. The state-space averaged open-loop model is given in Eq. (6). Input variables are considered to be the input voltage $v_{\mathrm{dc}}$ and the load current $i_{o}$. State variables are filter inductor current and capacitor voltage, $i_{\mathrm{L}}$ and $v_{\mathrm{C}}$, which are also output variable alongside with the current on the dcside, $i_{L}^{\mathrm{dc}}$. The load voltage is essentially the filter capacitor voltage. The control variable is the duty cycle $d^{\mathrm{HB}}$. The openloop dynamics can be solved using Eq. (2) and is represented by Eq. (7).

$$
\left[\begin{array}{c}
\tilde{\mathbf{i}}_{\mathrm{L}, \mathrm{dq}} \\
\tilde{\mathbf{v}}_{\mathrm{C}, \mathrm{dq}} \\
\tilde{i}_{L}^{\mathrm{dc}}
\end{array}\right]=\left[\begin{array}{ccc}
\mathbf{G}_{\mathrm{iL}, \mathrm{o}} & \mathbf{T}_{\mathrm{oL}, \mathrm{o}} & \mathbf{G}_{\mathrm{cL}, \mathrm{o}} \\
\mathbf{G}_{\mathrm{iC}, \mathrm{o}} & \mathbf{Z}_{\mathrm{out}, \mathrm{o}} & \mathbf{G}_{\mathrm{co}, \mathrm{o}} \\
\mathbf{Y}_{\mathrm{in}, \mathrm{o}} & \mathbf{T}_{\mathrm{oi}, \mathrm{o}} & \mathbf{G}_{\mathrm{ci}, \mathrm{o}}
\end{array}\right]\left[\begin{array}{c}
\tilde{v}_{\mathrm{dc}} \\
\tilde{\mathbf{i}}_{\mathrm{o}, \mathrm{dq}} \\
\tilde{\mathbf{d}}_{\mathrm{dq}}^{\mathrm{HB}}
\end{array}\right]
$$

The inverter control is performed as well in a cascaded manner in SRF. The inner loop controls the filter inductor current while the outer one controls the filter capacitor voltage, or the output voltage. Under the inductor closed-loop current control the control-to-output transfer function is given as

$$
\mathbf{G}_{\mathrm{co}, \mathrm{cl}}^{\mathrm{lcc}}=\mathbf{G}_{\mathrm{co}, \mathrm{o}}\left[\mathbf{I}-\left(\mathbf{G}_{\mathrm{PI}}^{\mathrm{i}}-\mathbf{G}_{\mathrm{dec}}^{\mathrm{i}}\right) \mathbf{H}_{\mathrm{i}} \mathbf{G}_{\mathrm{cL}, \mathrm{o}}\right]^{-1} \mathbf{G}_{\mathrm{PI}}^{\mathrm{i}}
$$

While under the capacitor voltage control loop close the control-to-output transfer function is given as

$$
\mathbf{G}_{\mathrm{co}, \mathrm{cl}}^{\mathrm{cvc}}=\mathbf{G}_{\mathrm{co}, \mathrm{cl}}^{\mathrm{lcc}}\left[\mathbf{I}-\left(\mathbf{G}_{\mathrm{PI}}^{\mathrm{v}}-\mathbf{G}_{\mathrm{dec}}^{\mathrm{v}}\right) \mathbf{H}_{\mathrm{v}} \mathbf{G}_{\mathrm{co}, \mathrm{cl}}^{\mathrm{lcc}}\right]^{-1} \mathbf{G}_{\mathrm{PI}}^{\mathrm{v}}
$$

Where $\mathbf{G}_{\mathrm{dec}}^{\mathrm{i}}, \mathbf{G}_{\mathrm{dec}}^{\mathrm{v}}, \mathbf{G}_{\mathrm{PI}}^{\mathrm{i}}$ and $\mathbf{G}_{\mathrm{PI}}^{\mathrm{v}}$ are the current and voltage controller decoupling matrices and current and voltage PI regulators respectively, while matrices $\mathbf{H}$ are current and voltage transducer matrices. Moreover, the closed-loop dynamics of 


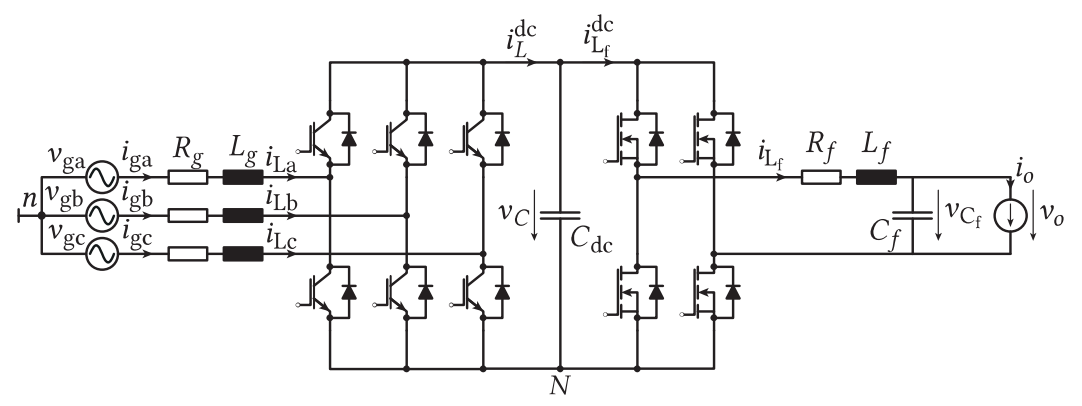

Fig. 2. Three-phase supplied Active Front End (AFE) interfaced to an HB phase inverter with an $L C$ filter.

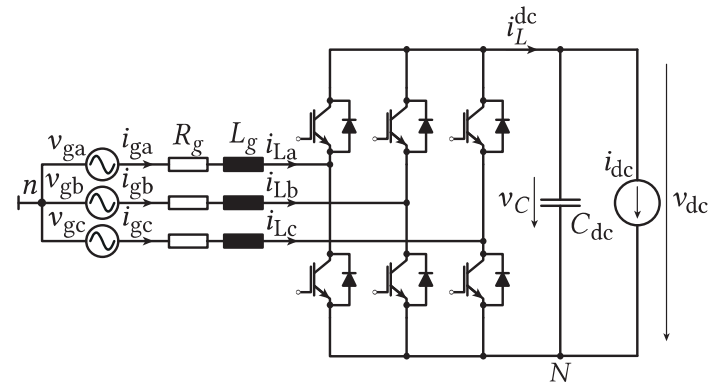

(a) Ideal three-phase current-fed AFE supplied from the secondaries of the MWT.

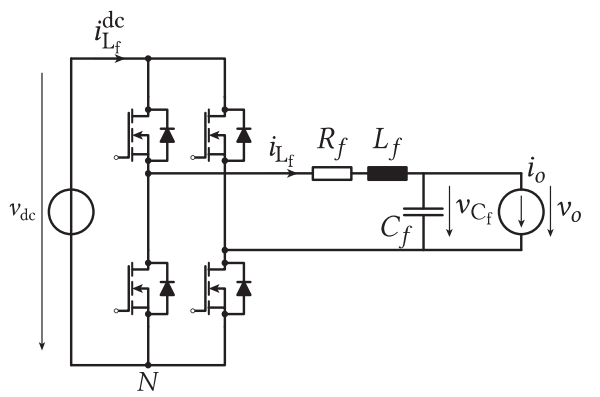

(b) HB with an output $L C$ filter.

Fig. 3. Partitioned CHB cell for input and output stage modelling.
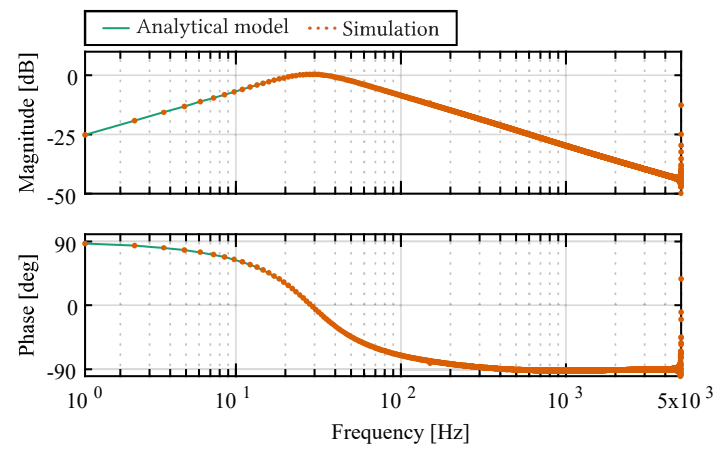

Fig. 4. Output impedance of the AFE under the input current and output voltage control loops closed. the HB-inverter can be represented using the block-diagram of Fig. 5

\section{SourCe-AFFected Dynamics Characterization}

In Section III-B it was stated that the dc-link voltage source is ideal with respect to the fact that the voltage was controlled on the AFE side. However, in practice the control loops do not have an infinite bandwidth and as such the voltage source cannot be considered ideal. This means that the source contains a non-zero finite internal impedance which is the closed loop output impedance $Z_{\text {out,cl }}^{\mathrm{dvc}}$ of the active input element, i.e. the AFE, as depicted in Fig. 6. From Fig. 6 the dc-side current of the inverter can be defined as in Eq. (10) and from Eq. (7) the $i_{\mathrm{dc}}^{L}$ can be obtained as in Eq. (11)

$$
\begin{gathered}
i_{\mathrm{dc}}^{L}=\frac{v_{\mathrm{dc}}^{\mathrm{s}}-v_{\mathrm{dc}}}{Z_{\mathrm{out}, \mathrm{cl}}^{\mathrm{dvc}}} \\
i_{\mathrm{dc}}^{L}=Y_{\mathrm{in}, \mathrm{cl}}^{\mathrm{cvc}} v_{\mathrm{dc}}+\mathbf{G}_{\mathrm{ci}, \mathrm{cl}}^{\mathrm{cvc}} \tilde{\mathbf{v}}_{\mathrm{C}, \mathrm{dq}}^{\star}+\mathbf{T}_{\mathrm{oi}, \mathrm{cl}}^{\mathrm{cvc}} \tilde{\mathbf{i}}_{\mathrm{o}, \mathrm{dq}}
\end{gathered}
$$

Plugging Eq. (10) into Eq. (11) gives the $v_{\mathrm{dc}}^{\mathrm{s}}$ expressed in terms of $v_{\mathrm{dc}}$ as

$$
\tilde{v}_{\mathrm{dc}}=\frac{\tilde{v}_{\mathrm{dc}}^{\mathrm{s}}-Z_{\mathrm{out}, \mathrm{cl}}^{\mathrm{dvc}} \mathbf{G}_{\mathrm{ci}, \mathrm{cl}}^{\mathrm{cvc}} \tilde{\mathbf{v}}_{\mathrm{C}, \mathrm{dq}}^{\star}-Z_{\mathrm{out}, \mathrm{cl}}^{\mathrm{dvc}} \mathbf{T}_{\mathrm{oi}, \mathrm{cl}}^{\mathrm{cvc}} \tilde{\mathbf{i}}_{\mathrm{o}, \mathrm{dq}}}{1+Z_{\mathrm{out}, \mathrm{cl}}^{\mathrm{dvc}} Y_{\mathrm{in}, \mathrm{cl}}^{\mathrm{cvc}}}
$$

Replacing $\tilde{v}_{\mathrm{dc}}$ in Eq. (7) by Eq. (12) gives the closed-loop source affected dynamics in Eq. (13).

\section{Single-Phase Measurements in $d q$-Frame}

The control-to-output characteristics $\mathbf{G}_{\mathrm{co}, \mathrm{cl}}^{\mathrm{cvc}}$ of the inverter in the $d q$-frame is a small-signal characteristic at the dc level while the actual single-phase system is an ac system and as such the characteristics cannot be directly measured in the $d q$ frame. Instead, an approach in which the measurements from a single-phase system are transferred into the $d q$-frame needs to be devised. For this purpose, a measurement and verification process based on creating two orthogonal systems is used. The first system is the $\alpha$ system while the orthogonal is the $\beta$ system created through converter control. 


$$
s\left[\begin{array}{c}
\tilde{i}_{\mathrm{Ld}} \\
\tilde{i}_{\mathrm{Lq}} \\
\tilde{v}_{\mathrm{Cd}} \\
\tilde{v}_{\mathrm{Cq}}
\end{array}\right]=\underbrace{\left[\begin{array}{cccc}
-\frac{R_{f}}{L_{f}} & \omega_{s} & -\frac{1}{L_{f}} & 0 \\
-\omega_{s} & -\frac{R_{f}}{L_{f}} & 0 & -\frac{1}{L_{f}} \\
\frac{1}{C_{f}} & 0 & 0 & \omega_{s} \\
0 & \frac{1}{C_{f}} & -\omega_{s} & 0
\end{array}\right]}_{\mathbf{A}}\left[\begin{array}{l}
{\left[\begin{array}{c}
\tilde{i}_{\mathrm{Ld}} \\
\tilde{i}_{\mathrm{Lq}} \\
\tilde{v}_{\mathrm{Cd}} \\
\tilde{v}_{\mathrm{Cq}}
\end{array}\right]+} \\
{\left[\begin{array}{ccccc}
-\frac{D_{d}^{\mathrm{HB}}}{L_{f}} & 0 & 0 & -\frac{V_{\mathrm{dc}}}{L_{f}} & 0 \\
-\frac{D_{q}^{\mathrm{HB}}}{L_{f}} & 0 & 0 & 0 & -\frac{V_{\mathrm{dc}}}{L_{f}} \\
0 & -\frac{1}{C_{f}} & 0 & 0 & 0 \\
0 & 0 & -\frac{1}{C_{f}} & 0 & 0
\end{array}\right]} \\
\mathbf{B}
\end{array}\right]\left[\begin{array}{c}
\tilde{v}_{\mathrm{dc}} \\
\tilde{i}_{\mathrm{od}} \\
\tilde{i}_{\mathrm{oq}} \\
\tilde{d}_{d}^{\mathrm{HB}} \\
\tilde{d}_{q}^{\mathrm{HB}}
\end{array}\right]
$$

$$
\left[\begin{array}{c}
\tilde{i}_{\mathrm{Ld}} \\
\tilde{i}_{\mathrm{Lq}} \\
\tilde{v}_{\mathrm{Cd}} \\
\tilde{v}_{\mathrm{Cq}} \\
\tilde{i}_{L}^{\mathrm{dc}}
\end{array}\right]=\underbrace{\left[\begin{array}{cccc}
1 & 0 & 0 & 0 \\
0 & 1 & 0 & 0 \\
0 & 0 & 1 & 0 \\
0 & 0 & 0 & 1 \\
D_{d}^{\mathrm{HB}} & D_{q}^{\mathrm{HB}} & 0 & 0
\end{array}\right]}_{\mathbf{C}}\left[\begin{array}{c}
\tilde{i}_{\mathrm{Ld}} \\
\tilde{i}_{\mathrm{Lq}} \\
\tilde{v}_{\mathrm{Cd}} \\
\tilde{v}_{\mathrm{Cq}}
\end{array}\right]+\underbrace{\left[\begin{array}{ccccc}
0 & 0 & 0 & 0 & 0 \\
0 & 0 & 0 & 0 & 0 \\
0 & 0 & 0 & 0 & 0 \\
0 & 0 & 0 & 0 & 0 \\
0 & 0 & 0 & I_{\mathrm{Ld}} & I_{\mathrm{Lq}}
\end{array}\right]}_{\mathbf{D}}\left[\begin{array}{c}
\tilde{v}_{\mathrm{dc}} \\
\tilde{i}_{\mathrm{od}} \\
\tilde{i}_{\mathrm{oq}} \\
\tilde{d}_{d}^{\mathrm{HB}} \\
\tilde{d}_{q}^{\mathrm{HB}}
\end{array}\right]
$$

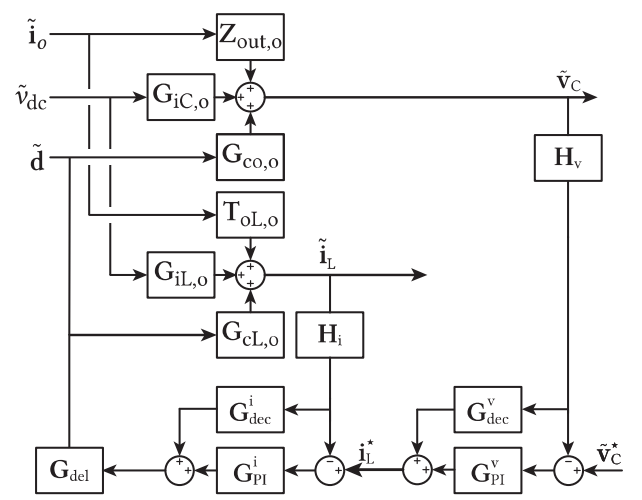

Fig. 5. Closed-loop dynamics of the HB inverter with cascaded inductor current and capacitor voltage control.

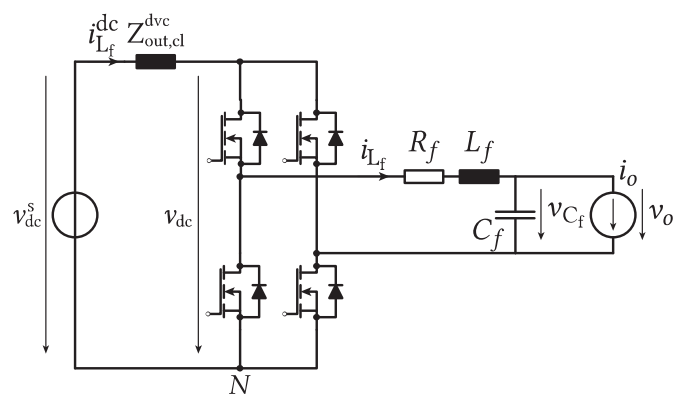

Fig. 6. HB with an output LC filter and internal source impedance.

\section{A. Control-to-Output Characteristics}

The $\mathbf{G}_{\mathrm{co}, \mathrm{cl}}^{\mathrm{cvc}}$ is defined as

$$
\left[\begin{array}{c}
\tilde{v}_{\mathrm{Cd}} \\
\tilde{v}_{\mathrm{Cq}}
\end{array}\right]=\mathbf{G}_{\mathrm{co}, \mathrm{cl}}^{\mathrm{cvc}}\left[\begin{array}{l}
\tilde{v}_{\mathrm{Cd}}^{\star} \\
\tilde{v}_{\mathrm{Cq}}^{\star}
\end{array}\right]
$$

To extract the frequency response of $\mathbf{G}_{\mathrm{co}, \mathrm{cl}}^{\mathrm{cvc}}$, the process needs to be performed with two sets of voltage measurements, and thus it can be uniquely determined. $\mathbf{G}_{\mathrm{co}, \mathrm{cl}}^{\mathrm{cvc}}$ can be calculated as

$$
\mathbf{G}_{\mathrm{co}, \mathrm{cl}}^{\mathrm{cvc}}=\left[\begin{array}{ll}
\tilde{v}_{\mathrm{Cd}, 1} & \tilde{v}_{\mathrm{Cd}, 2} \\
\tilde{v}_{\mathrm{Cq}, 1} & \tilde{v}_{\mathrm{Cq}, 2}
\end{array}\right]\left[\begin{array}{ll}
\tilde{v}_{\mathrm{Cd}, 1}^{\star} & \tilde{v}_{\mathrm{Cd}, 2}^{\star} \\
\tilde{v}_{\mathrm{Cq}, 1}^{\star} & \tilde{v}_{\mathrm{Cd}, 2}^{\star}
\end{array}\right]^{-1}
$$

Moreover, $\left[\tilde{v}_{\mathrm{Cd}, 1}, \tilde{v}_{\mathrm{Cq}, 1}\right]$ and $\left[\tilde{v}_{\mathrm{Cd}, 2}, \tilde{v}_{\mathrm{Cq}, 2}\right]$ are linearly independent as well as $\left[\tilde{v}_{\mathrm{Cd}, 1}^{\star}, \tilde{v}_{\mathrm{Cq}, 1}^{\star}\right]$ and $\left[\tilde{v}_{\mathrm{Cd}, 2}^{\star}, \tilde{v}_{\mathrm{Cq}, 2}^{\star}\right]$. The perturbation can be injected directly in the reference voltage $\tilde{\mathbf{v}}_{\mathrm{C}, \mathrm{dq}}^{\star}$ in the control system, but the response can only be measured on the inverter terminals in the single-phase system.

\section{B. Wideband Perturbation Injection}

An extensively used method in system identification measurements is the sine sweep method requiring one by one frequency small-signal perturbation injection and collection of responses, thus requiring large number of measurements and time to be executed. If the measurement time is too long, the measured object may as well be disturbed and change its operating point and subsequently the characteristics measured would change. This is somewhat improved with multi-tone signals but the energy of this signal is reduced with the number of tones it consists of. Lately, the PRBS signals (cf. Fig. 7) have seen substantial use for system identification and impedance measurement [12]-[14]. The advantage of these signals lies in the fact that they are well suited for the characterization of dynamics systems where rapid measurement is necessary due to the possibility of variation of the system state in time. What is common for measurements in [12]-[14] is that they are performed in dc or three-phase ac systems after which the results are represented in $d q$-frame even though they represent ac measurements. Here, a method that employs the wideband PRBS signal for the control-tooutput characteristics of a single-phase inverter in the $d q$ frame is presented. Measurement process of $\mathbf{G}_{\mathrm{co}, \mathrm{cl}}^{\mathrm{cvc}}$ requires two perturbation injections and two measurement sets. First perturbation is injected into the $d$-axis voltage reference, while 


$$
\left[\begin{array}{c}
\tilde{\mathbf{i}}_{\mathrm{L}, \mathrm{dq}} \\
\tilde{\mathbf{v}}_{\mathrm{C}, \mathrm{dq}} \\
\tilde{i}_{L}^{\mathrm{dc}}
\end{array}\right]=\left[\begin{array}{ccc}
\frac{\mathbf{G}_{\mathrm{i}, \mathrm{cl}}^{\mathrm{cvc}}}{1+Z_{\mathrm{out}, \mathrm{cl}}^{\mathrm{dvc}} Y_{\mathrm{in}, \mathrm{cl}}^{\mathrm{cvc}}} & \mathbf{T}_{\mathrm{oL}, \mathrm{cl}}^{\mathrm{cvc}}-\frac{\mathbf{G}_{\mathrm{iL}, \mathrm{cl}}^{\mathrm{cvc}} Z_{\mathrm{out}, \mathrm{cl}}^{\mathrm{dvc}} \mathbf{T}_{\mathrm{o}, \mathrm{cl}}^{\mathrm{cvc}}}{1+Z_{\mathrm{out}, \mathrm{cl}}^{\mathrm{dvc}} Y_{\mathrm{in}, \mathrm{cl}}^{\mathrm{cvc}}} & \mathbf{G}_{\mathrm{cL}, \mathrm{cl}}^{\mathrm{cvc}}-\frac{\mathbf{G}_{\mathrm{iL}, \mathrm{cl}}^{\mathrm{cvc}} Z_{\mathrm{out}, \mathrm{cl}}^{\mathrm{dvc}} \mathbf{G}_{\mathrm{ci}, \mathrm{cl}}^{\mathrm{cvc}}}{1+Z_{\mathrm{out}, \mathrm{cl}}^{\mathrm{dvc}} Y_{\mathrm{in}, \mathrm{cl}}^{\mathrm{cv}}} \\
\frac{\mathbf{G}_{\mathrm{iC}, \mathrm{cl}}^{\mathrm{cvc}}}{1+Z_{\mathrm{out}, \mathrm{cl}}^{\mathrm{dvc}} Y_{\mathrm{in}, \mathrm{cl}}^{\mathrm{cvc}}} & \mathbf{Z}_{\mathrm{out}, \mathrm{cl}}^{\mathrm{cvc}}-\frac{\mathbf{G}_{\mathrm{iL}, \mathrm{cl}}^{\mathrm{cvc}} Z_{\mathrm{out}, \mathrm{cl}}^{\mathrm{dvc}} \mathbf{T}_{\mathrm{oi}, \mathrm{cl}}^{\mathrm{cvc}}}{1+Z_{\mathrm{out}, \mathrm{cl}}^{\mathrm{dvc}} Y_{\mathrm{in}, \mathrm{cl}}^{\mathrm{cvc}}} & \mathbf{G}_{\mathrm{co}, \mathrm{cl}}^{\mathrm{cvc}}-\frac{\mathbf{G}_{\mathrm{iC}, \mathrm{cl}}^{\mathrm{cvc}} Z_{\mathrm{out}, \mathrm{cl}}^{\mathrm{dvc}} \mathbf{G}_{\mathrm{ci}, \mathrm{cl}}^{\mathrm{cvc}}}{1+Z_{\mathrm{out}, \mathrm{cl}}^{\mathrm{dvc}} Y_{\mathrm{in}, \mathrm{cl}}^{\mathrm{cvc}}} \\
\frac{Y_{\mathrm{in}, \mathrm{cl}}^{\mathrm{cvc}}}{1+Z_{\mathrm{out}, \mathrm{cl}}^{\mathrm{dvc}} Y_{\mathrm{in}, \mathrm{cl}}^{\mathrm{cvc}}} & \frac{\mathbf{T}_{\mathrm{oi}, \mathrm{cl}}^{\mathrm{cvc}}}{1+Z_{\mathrm{out}, \mathrm{cl}}^{\mathrm{dvc}} Y_{\mathrm{in}, \mathrm{cl}}^{\mathrm{cvc}}} & \frac{\mathbf{G}_{\mathrm{ci}, \mathrm{cl}}^{\mathrm{cvc}}}{1+Z_{\mathrm{out}, \mathrm{cl}}^{\mathrm{dvc}} Y_{\mathrm{in}, \mathrm{cl}}^{\mathrm{cvc}}}
\end{array}\right]\left[\begin{array}{c}
\tilde{v}_{\mathrm{dc}}^{\mathrm{s}} \\
\tilde{\mathbf{i}}_{\mathrm{O}, \mathrm{dq}} \\
\tilde{\mathbf{v}}_{\mathrm{C}, \mathrm{dq}}^{\star}
\end{array}\right]
$$
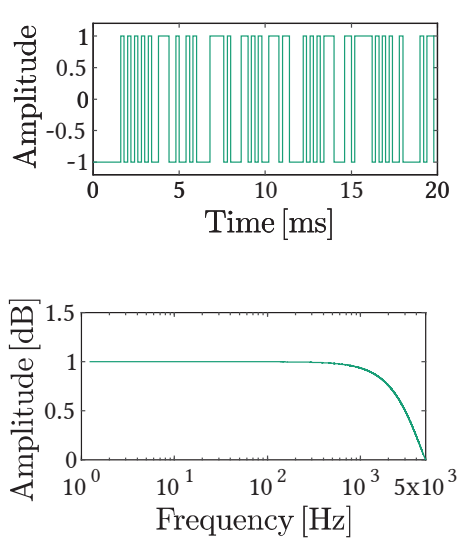

Fig. 7. PRBS-12 signal in time and frequency domain

the $q$-axis perturbation is kept at zero value. For the second injection, the opposite holds. Thus the perturbation matrix $\mathbf{P}(t)$ in the $d q$-frame is designed as

$$
\mathbf{P}(t)=\left[\begin{array}{ll}
v_{\mathrm{pd}, 1} & v_{\mathrm{pd}, 2} \\
v_{\mathrm{pq}, 1} & v_{\mathrm{pq}, 2}
\end{array}\right]=\left[\begin{array}{cc}
p(t) & 0 \\
0 & p(t)
\end{array}\right]
$$

where the $p(t)$ is the PRBS signal in time domain, and the perturbation is injected into the voltage reference $\tilde{\mathbf{v}}_{\mathrm{C}, \mathrm{dq}}^{\star}$

The idea behind the single-phase measurement of $\mathbf{G}_{\mathrm{co}, \mathrm{cl}}^{\mathrm{cvc}}$ in $d q$-frame lies in measuring the response of two systems, one original and one orthogonal to it. For the original system, the Park transform and its inverse are given as:

$$
\begin{aligned}
& \mathbf{T}_{\Theta}=\left[\begin{array}{cc}
\cos \theta_{s} & \sin \theta_{s} \\
-\sin \theta_{s} & \cos \theta_{s}
\end{array}\right] \\
& \mathbf{T}_{\Theta}^{-1}=\left[\begin{array}{cc}
\cos \theta_{s} & -\sin \theta_{s} \\
\sin \theta_{s} & \cos \theta_{s}
\end{array}\right]
\end{aligned}
$$

While for the orthogonal system all the transformations and its inverse in the control algorithm are rotated by $\frac{\pi}{2}$ and are given as:

$\overline{\mathbf{T}}_{\Theta}=\left[\begin{array}{cc}\cos \left(\theta_{s}-\frac{\pi}{2}\right) & \sin \left(\theta_{s}-\frac{\pi}{2}\right) \\ -\sin \left(\theta_{s}-\frac{\pi}{2}\right) & \cos \left(\theta_{s}-\frac{\pi}{2}\right)\end{array}\right]=\left[\begin{array}{cc}\sin \theta_{s} & -\cos \theta_{s} \\ \cos \theta_{s} & \sin \theta_{s}\end{array}\right]$ $\overline{\mathbf{T}}_{\Theta}^{-1}=\left[\begin{array}{cc}\sin \theta_{s} & \cos \theta_{s} \\ -\cos \theta_{s} & \sin \theta_{s}\end{array}\right]$

where $\theta_{s}=\omega_{s} t$. For this approach to be possible it is considered that one has the access to the control system, to which the authors of this work as the system designers

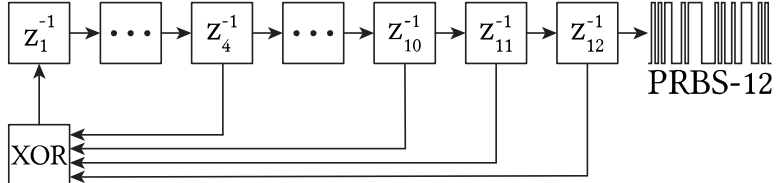

Fig. 8. Shift register circuit for PRBS generation

have, since the ultimate goal is to build an MV-CHB for perturbation injection and impedance/admittance measurement. After injecting the perturbation and measuring the output voltages, the original system will provide the voltage $v_{C, \alpha}$, while the orthogonal one will provide $v_{C, \beta}$. The two voltage measurements are then combined and transformed into their $d q$-frame counterparts, thus effectively providing a way to perform single-phase measurements in $d q$-frame. Once the measurements are obtained one needs to extract the frequency spectrum of the measurements using discrete Fourier Transform (DFT) algorithm upon which the equation Eq. (15) can be applied.

\section{Simulation RESUlts}

The performance of the proposed modelling and measurement method is put under test using PLECS simulation environment. A $5 \mathrm{kHz}$ PRBS-12 signal is injected into the output voltage reference in the converter control system and the resulting output voltage is measured. In order to investigate the effect of presence of an active element in the cell, two types of $\mathrm{CHB}$ cell were simulated. The first cell type is a standalone HB-inverter with an ideal voltage source as an input, while in the second case the AFE control system controls the capacitor dc-link voltage which serves as an input voltage source to the HB-inverter. In both cases, the HB-inverter is operating with parameter give in Table II. The set of result is provided in Fig. 9 where the $d$ - and $q$-axis control-to-output characteristics, $\mathbf{G}_{\mathrm{co}, \mathrm{cl}}^{\mathrm{cvc}}$, of the HB-inverter are given. From the presented frequency response it is noticeable there is either slight or no difference between the analytically predicted control-to-output characteristics and the simulation measurements. Moreover, by comparing Figs. 9a and 9c with Figs. 9b and 9d it is observed that the presence of the AFE on the input side does not lead to limitation on the output dynamics of the HB-inverter and that the two are perfectly decoupled. These results permit to continue future impedance / admittance measurement analysis using CHB converter without being concerned by the impact of the AFE on the output of the CHB. 

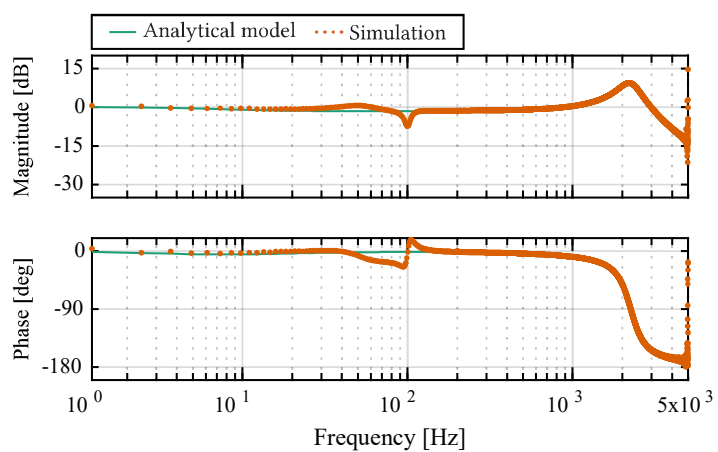

(a)
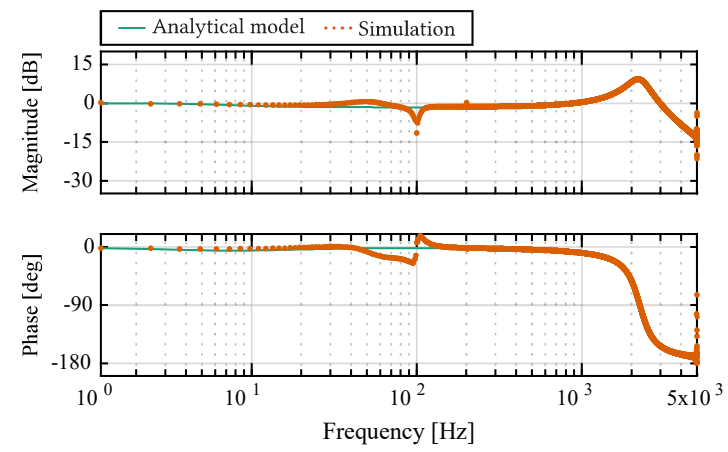

(c)
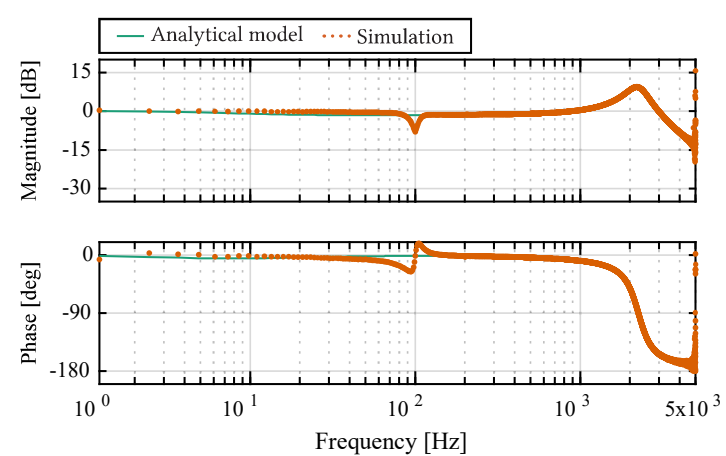

(b)
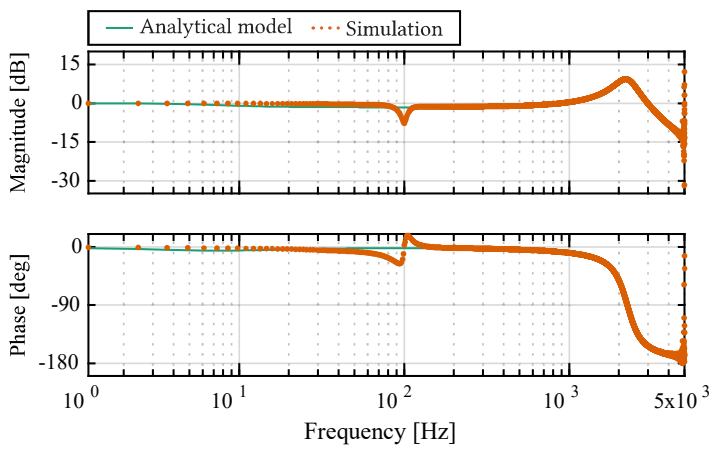

(d)

Fig. 9. a) $d$-axis and c) $q$-axis control-to-output characteristics $\mathbf{G}_{\mathrm{co}, \mathrm{cl}, \mathrm{dd}}^{\mathrm{cvc}}$ and $\mathbf{G}_{\mathrm{co}, \mathrm{cl}, \mathrm{qq}}^{\mathrm{cvc}}$ with the standalone-operation of the HB-inverter compared to the same characteristics, b) and d), when the AFE is operating at the input stage.

\section{CONCLUSION}

This paper presented a promising solution for the problem of MV impedance/admittance measurement based on the MVCHB converter. To confirm the suitability of the proposed idea, the model of the converter is developed systematically starting with the model of one single-phase cell of the converter and by confirming that the AFE input stage of a single cell does not affect the HB output stage dynamics. The conclusion is reached through the wideband measurement of the output dynamics using PRBS signals, while the results are shown methodically through analytical modelling and simulations. This type of measurement can successfully be performed in $d q$-frame by combining two orthogonal single-phase systems whose response is represented in the $d q$-frame. Compared to previous measurement methods, such as the much slower ac-sweep method, the wideband measurements allow rapid verification of concepts put forward. Moreover, the use of wideband measurements, namely the use of PRBS signals, reduce the risk of the measured system changing state while being measured. These, first results are vital for the development of the full scale converter as it confirms its viability in terms of the output voltage quality and control bandwidth.

\section{REFERENCES}

[1] X. Wang and F. Blaabjerg, "Harmonic stability in power electronic based power systems: Concept, modeling, and analysis," IEEE Transactions on Smart Grid, pp. 11,2018

[2] U. Javaid, F. D. Freijedo, D. Dujic, and W. Van Der Merwe, "Dynamic assessment of source-load interactions in marine MVDC distribution," IEEE Transactions on Industrial Electronics, vol. 64, no. 6, pp. 4372-4381, 2017.

[3] A. Riccobono and E. Santi, "Comprehensive review of stability criteria for dc power distribution systems," IEEE Transactions on Industry Applications, vol. 50, no. 5, pp. 3525-3535, Sep. 2014.

[4] U. Javaid, F. D. Freijedo, W. van der Merwe, and D. Dujic, "Stability analysis of multi-port mvdc distribution networks for all-electric ships," IEEE Journal of Emerging and Selected Topics in Power Electronics, pp. 1-1, 2019.

[5] M. Mogorovic and D. Dujic, "100 kw, $10 \mathrm{khz}$ mediumfrequency transformer design optimization and experimental verification," IEEE Transactions on Power Electronics, vol. 34, no. 2, pp. 1696-1708, Feb. 2019.

[6] M. Utvic, S. Milovanovic, and D. Dujic, "Flexible medium voltage dc source utilizing series connected modular multilevel converters," in [Detailed technical programme of the 21th European Conference on Power Electronics and Applications (EPE ECCE Europe)], 2019. 
[7] M. Petković, N. Hildebrandt, F. D. Freijedo, and D. Dujić, "Cascaded H-Bridge Multilevel Converter for a High-Power Medium-Voltage Impedance-Admittance Measurement Unit," in 2018 International Symposium on Industrial Electronics (INDEL), Nov. 2018, pp. 1-8.

[8] Y. Li, Y. Wang, R. Wang, J. Wu, H. Zhang, Y. Feng, S. Li, W. Yao, and B. Q. Li, "Distributed Generation Grid-Connected Converter Testing Device Based on Cascaded H-Bridge Topology," IEEE Transactions on Industrial Electronics, vol. 63, no. 4, pp. 2143-2154, Apr. 2016.

[9] R. W. Erickson and D. Maksimovic, Fundamentals of power electronics. Springer Science \& Business Media, 2007.

[10] T. Suntio, T. Messo, and J. Puukko, Power Electronic Converters: Dynamics and Control in Conventional and Renewable Energy Applications. Wiley, 2017.

[11] V. Blasko and V. Kaura, "A new mathematical model and control of a three-phase AC-DC voltage source converter," IEEE Transactions on Power Electronics, vol. 12, no. 1, pp. 116-123, Jan. 1997.

[12] R. Luhtala, T. Roinila, and T. Messo, "Implementation of real-time impedance-based stability assessment of grid-connected systems using mimo-identification techniques," IEEE Transactions on Industry Applications, vol. 54, no. 5, pp. 5054-5063, Sep. 2018.

[13] T. Roinila, H. Abdollahi, S. Arrua, and E. Santi, "Realtime stability analysis and control of multiconverter systems by using mimo-identification techniques," IEEE Transactions on Power Electronics, vol. 34, no. 4, pp. 3948-3957, Apr. 2019.

[14] T. Roinila, T. Messo, R. Luhtala, R. Scharrenberg, E. C. W. de Jong, A. Fabian, and Y. Sun, "Hardwarein-the-loop methods for real-time frequency-response measurements of on-board power distribution systems," IEEE Transactions on Industrial Electronics, vol. 66, no. 7, pp. 5769-5777, Jul. 2019. 